\title{
Finite Element Simulation of Hydrogen Dispersion by the Analogy of the Boussinesq Approximation*
}

\author{
Hiroshi KANAYAMA ${ }^{* *}$, Hisayoshi TSUKIKAWA ${ }^{* * *}$, \\ Stephane Boris NDONG-MEFANE ${ }^{* * *}$ and Osamu SAKURAGI ${ }^{* * *}$ \\ **Department of Mechanical Engineering, Faculty of Engineering, Kyushu University, \\ Motooka 744, Nishi-ku, Fukuoka, Japan 819-0395 \\ E-mail: kanayama@mech.kyushu-u.ac.jp \\ ${ }^{* \star *}$ Department of Intelligent Machinery and Systems, Graduate School of Engineering, \\ Kyushu University, \\ Motooka 744, Nishi-ku, Fukuoka, Japan 819-0395
}

\begin{abstract}
Hydrogen is expected as new fuel instead of fossil fuel. It will be used as fuel of a fuel cell for which development is performed actively. But it is difficult to experiment the hydrogen dispersion in case of hydrogen leaks. Therefore clarifying the hydrogen dispersion with numerical analysis becomes important. Furthermore, hydrogen dispersion under various conditions can be clarified with numerical analysis, which is useful to use hydrogen safely. This paper deals with computer simulation of the hydrogen dispersion by a finite element method. The mathematical model of hydrogen dispersion is governed by the momentum equations, the continuity equation and the hydrogen mass conservation equation. The model presented here is a three-dimensional, incompressible, non-stationary model. This paper describes a finite element method with the stabilization technique for solving Navier-Stokes equations and the advection diffusion equation for hydrogen concentration like the Boussinesq approximation of thermal convection problems. Improved numerical results are also shown.
\end{abstract}

Key words: Hydrogen, Dispersion, Finite Element Method, Stabilization Method

\section{Introduction}

While various new energies are considered instead of fossil fuel, hydrogen is one of the most interesting energy in this century. It is greatly expanding the possibility from use as a current industrial gas to the basic energy of a social system. It will be used as fuel of a fuel cell for which development is performed actively. A fuel cell immediately changes the chemical energy of hydrogen and oxygen to electrical energy. This conversion has high efficiency and low emissions. In the demand for the zero emission and the $\mathrm{CO}_{2}$ reduction, the expectation for clean hydrogen is growing more and more. Many scientists are studying characteristic features of hydrogen. But it is difficult to experiment the hydrogen dispersion in case of hydrogen leaks, because hydrogen has a high diffusion coefficient and low kinematic viscosity. Hence clarifying the hydrogen dispersion with numerical analysis becomes important ${ }^{1}$. 
This paper demonstrates a simulation of the hydrogen dispersion by a finite element method with the stabilization technique ${ }^{2)}$. In the previous paper ${ }^{3)}$, we used the same method but numerical results did not agree with experimental ones. By changing boundary conditions suitably, this paper shows improved results. The organization of the paper is as follows. In Section 2, a three-dimensional, incompressible, non-stationary model is developed to simulate the hydrogen dispersion. The equations consist of the momentum equations, the continuity equation and the hydrogen mass conservation equation. Section 3 describes a finite element method with the stabilization technique for solving Navier-Stokes equations and the advection diffusion equation for hydrogen concentration like the Boussinesq approximation of thermal convection problems ${ }^{4)}$. We use Bercovier-Pironneau elements ${ }^{5)}$ for the velocity and the pressure, and smaller P1 elements for the concentration of hydrogen. The backward Euler method is used for the time integration. In Section 4, numerical results are reported. Finally conclusions are described in Section 5.

\section{A Mathematical Model}

A three-dimensional incompressible non-stationary model of hydrogen dispersion is developed to simulate the hydrodynamics of gas flow. Figure 1 shows a computational model of a hallway ${ }^{1) 3}$. The hydrogen leaks from the floor at the left end of the hallway. There are a roof vent and a lower door vent for the gas ventilation at the right end of the hallway. The shapes of the hydrogen inlet and two vents are rectangles like Fig.1. Four sensors were set at black points in Fig.1.

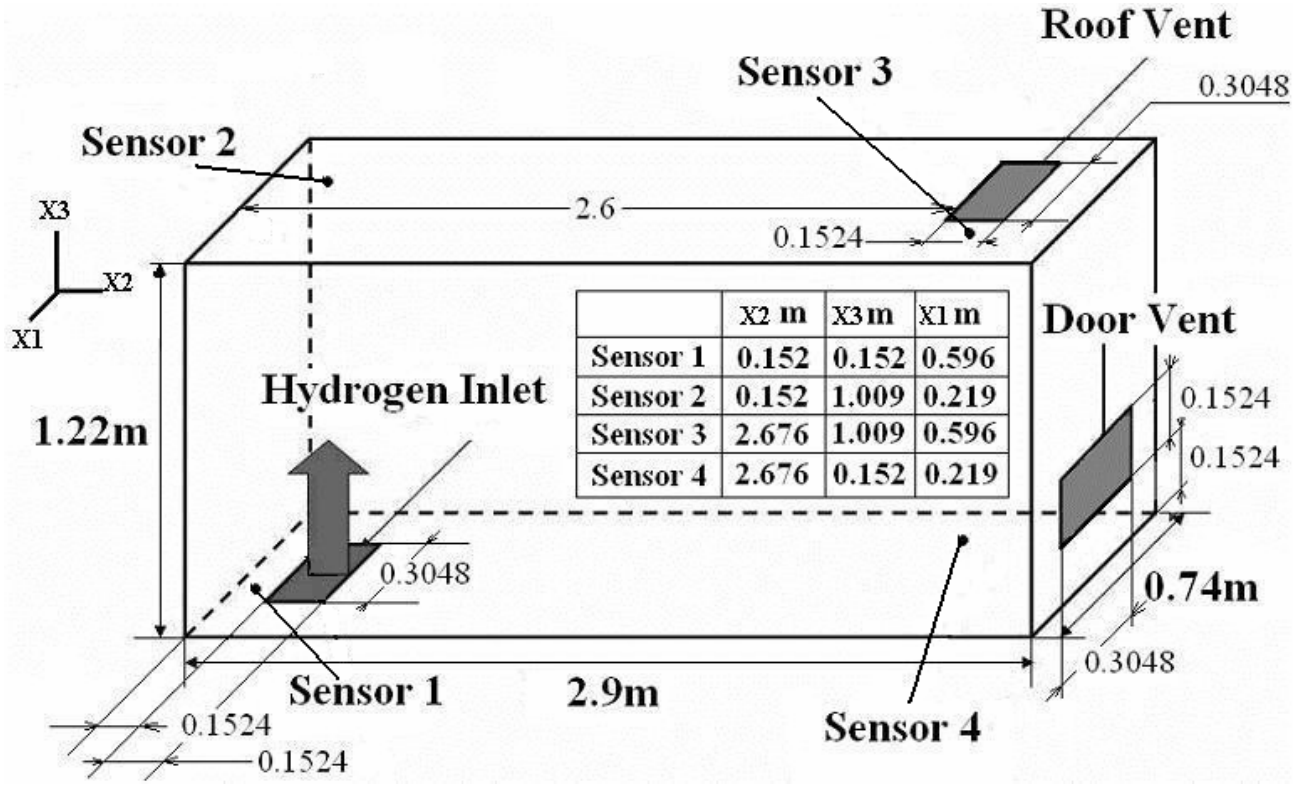

Fig.1 The ventilation model.

\section{$2.1 \quad$ Basic Equations}

Let $\Omega$ be the three-dimensional polyhedral domain with the boundary $\partial \Omega$. The conservation equations of mass and momentum are as follows; 


$$
\left\{\begin{array}{l}
\nabla \cdot \mathbf{u}=0 \\
\frac{\partial \mathbf{u}}{\partial t}+(\mathbf{u} \cdot \nabla) \mathbf{u}-2 v \nabla \cdot D(\mathbf{u})+\nabla p=-\beta C \mathbf{g}
\end{array}\right.
$$

The mass conservation equation for the hydrogen is written as

$$
\frac{\partial C}{\partial t}+\mathbf{u} \cdot \nabla C-a \Delta C=S
$$

In the above, $\mathbf{u}=\left(u_{1}, u_{2}, u_{3}\right)^{T}$ is the velocity $[\mathrm{m} / \mathrm{s}] ; t$ is time $[\mathrm{s}] ; v$ is the kinematic viscosity coefficient $\left[\mathrm{m}^{2} / \mathrm{s}\right] ; p$ is the gas mixture gauge pressure normalized by the density $\left[\mathrm{m}^{2} / \mathrm{s}^{2}\right]$ ( hereafter $p$ is shortly called pressure ) ; $\mathbf{g}=\left(g_{1}, g_{2}, g_{3}\right)^{T}$ is the gravity $\left[\mathrm{m} / \mathrm{s}^{2}\right] ; \beta$ is the coefficient [-]; $C$ is the mass concentration of hydrogen [mass $\left.\%\right] ; a$ is the hydrogen diffusion coefficient in air $\left[\mathrm{m}^{2} / \mathrm{s}\right] ; S$ is the source term $[1 / \mathrm{s}]$; and $D_{i j}$ is the rate of strain tensor $[1 / \mathrm{s}]$ defied by

$$
D_{i j}(\mathbf{u}) \equiv \frac{1}{2}\left(\frac{\partial u_{i}}{\partial x_{j}}+\frac{\partial u_{j}}{\partial x_{i}}\right) \quad i, j=1,2,3 .
$$

\subsection{Boundary Conditions}

Here, the boundary conditions are described precisely. $\Gamma_{\text {inlet }}, \Gamma_{\text {roof }}$ and $\Gamma_{\text {door }}$ denote the boundary of the hydrogen inlet, the boundary of the roof vent, and the boundary of the door vent, respectively.

At the inlet, the hydrogen leaks in the vertical direction. The velocity and the concentration are specified as follows;

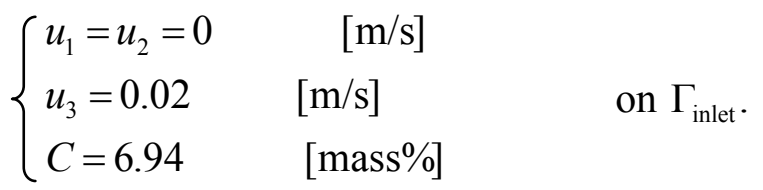

It is noted that the mass concentration is set to be 6.94 [mass\%] by considering the density difference between air and hydrogen. The Reynolds number on the inlet is below 60 .

Boundary conditions of both vents are the following. At the roof vent, the hydrogen is discharged outside freely. However, at the door vent, the air comes from the boundary because the inflow condition appears in this model.

$$
\begin{cases}\sum_{j=1}^{3} \sigma_{i j} n_{j}=0 \quad\left[\mathrm{~m}^{2} / \mathrm{s}^{2}\right] & \\ a \frac{\partial C}{\partial n}=0[\mathrm{~m} / \mathrm{s}] & \text { on } \Gamma_{\text {roof }},\end{cases}
$$




$$
\left\{\begin{array}{ll}
\sum_{j=1}^{3} \sigma_{i j} n_{j}=0 & {\left[\mathrm{~m}^{2} / \mathrm{s}^{2}\right]} \\
C=0 & {[\mathrm{mass} \%]}
\end{array} \quad \text { on } \Gamma_{\mathrm{door}},\right.
$$

where $\sigma(\mathbf{u}, p)$ is the stress tensor normalized by the density $\left[\mathrm{m}^{2} / \mathrm{s}^{2}\right]$ defined by

$$
\sigma_{i j}=-p \delta_{i j}+2 v D_{i j}(\mathbf{u}) \quad i, j=1,2,3 \text {, }
$$

with the Kronecker delta $\delta_{i j}$ and $n$ is the unit normal vector.

At the other boundary, there is no inflow of hydrogen.

$$
\left\{\begin{array}{l}
u_{1}=u_{2}=u_{3}=0[\mathrm{~m} / \mathrm{s}] \\
a \frac{\partial C}{\partial n}=0[\mathrm{~m} / \mathrm{s}]
\end{array} \quad \text { on } \partial \Omega-\left(\Gamma_{\text {inlet }}+\Gamma_{\text {roof }}+\Gamma_{\text {door }}\right) .\right.
$$

\subsection{Initial Conditions}

The initial conditions are described as follows;

$\left\{\begin{array}{ll}u_{1}=u_{2}=u_{3}= & 0 \quad[\mathrm{~m} / \mathrm{s}] \\ C=0 & {[\text { mass } \%]}\end{array} \quad\right.$ in $\Omega$.

\section{Numerical Method}

\subsection{Formulation}

Again, let $\Omega$ be a three-dimensional polyhedral domain with the boundary $\partial \Omega$.

$\Gamma_{u}$ denotes the boundary with specified velocity. $\Gamma_{c}$ denotes the boundary with specified concentration. We consider the non-stationary Navier-Stokes equations and the advection diffusion equation as

follows;

$$
\begin{array}{ll}
\frac{\partial \mathbf{u}}{\partial t}+(\mathbf{u} \cdot \nabla) \mathbf{u}-2 \nu \nabla \cdot D(\mathbf{u})+\nabla p & \\
=-\beta C \mathbf{g} & \text { in } \Omega \times(0, T), \\
\nabla \cdot \mathbf{u}=0 & \text { in } \Omega \times(0, T),
\end{array}
$$




$$
\begin{array}{lr}
\frac{\partial C}{\partial t}+\mathbf{u} \cdot \nabla C-a \Delta C=S & \text { in } \Omega \times(0, T), \\
\mathbf{u}=\hat{\mathbf{u}} & \text { on } \Gamma_{u} \times(0, T), \\
C=\hat{C} & \text { on } \Gamma_{c} \times(0, T), \\
\sum_{j=1}^{3} \sigma_{i j} n_{j}=0 & \text { on }\left(\partial \Omega-\Gamma_{u}\right) \times(0, T), \\
a \frac{\partial C}{\partial n}=0 & \text { on }\left(\partial \Omega-\Gamma_{c}\right) \times(0, T), \\
\mathbf{u}=\mathbf{u}^{0}, C=C^{0} & \text { in } \Omega \text { at } \mathrm{t}=0,
\end{array}
$$

where $T$ is the total time $[\mathrm{s}] ; \mathbf{u}^{0}$ is the initial velocity $[\mathrm{m} / \mathrm{s}] ; C^{0}$ is the initial concentration [mass\%]; $\hat{\mathbf{u}}$ is the boundary velocity $[\mathrm{m} / \mathrm{s}]$; and $\hat{C}$ is the boundary concentration [mass $\%]$.

As the weak form, the following system is considered;

$$
\begin{array}{ll}
\left(\frac{\partial \mathbf{u}}{\partial t}, \mathbf{v}\right)+((\mathbf{u} \cdot \nabla) \mathbf{u}, \mathbf{v})+(2 v D(\mathbf{u}), D(\mathbf{v}))-(p, \nabla \cdot \mathbf{v})+(\beta C \mathbf{g}, \mathbf{v})=0 & \text { for } \mathbf{v} \in V, \\
-(\nabla \cdot \mathbf{u}, q)=0 \quad \text { for } q \in Q, & \text { for } \theta \in \Theta .
\end{array}
$$

Here, $L^{2}(\Omega)$ denotes the space of square summable functions in $\Omega$, and $H^{1}(\Omega)$ is the space of functions in $L^{2}(\Omega)$ with derivatives up to the first order.

$$
\begin{aligned}
& V\left(\mathrm{~g}_{1}\right) \equiv\left\{\mathbf{v} \in\left(H^{1}(\Omega)\right)^{3} ; \mathbf{v}=\mathrm{g}_{1} \text { on } \Gamma_{u}\right\}, V \equiv V(0), \\
& Q \equiv\left\{q \in L^{2}(\Omega)\right\}, \\
& \Theta\left(g_{2}\right) \equiv\left\{\theta \in H^{1}(\Omega) ; \theta=g_{2} \text { on } \Gamma_{c}\right\}, \Theta \equiv \Theta(0),
\end{aligned}
$$

where $(\cdot, \cdot)$ denotes the $L^{2}$ - inner product over $\Omega$. 


\subsection{Finite Element Approximation}

Let us consider approximations of above formulations. The finite element method is used for discretization of space, and the backward Euler method is used for discretization of time. The Bercovier-Pironneau tetrahedral elements are used for approximations of velocity and pressure. The concentration is approximated like the velocity. The approximation scheme without stabilization terms is first explained as follows;

$$
\begin{aligned}
& \left(\frac{\mathbf{u}_{h}^{(n+1)}-\mathbf{u}_{h}^{(n)}}{\Delta t}, \mathbf{v}_{h}\right)+\left(\left(\mathbf{u}_{h}^{(n)} \cdot \nabla\right) \mathbf{u}_{h}^{(n+1)}, \mathbf{v}_{h}\right)+\left(2 v D\left(\mathbf{u}_{h}^{(n+1)}\right), D\left(\mathbf{v}_{h}\right)\right)-\left(p_{h}^{(n+1)}, \nabla \cdot \mathbf{v}_{h}\right) \\
& +\left(\beta C_{h}^{(n+1)} \mathbf{g}, \mathbf{v}_{h}\right)-\left(\nabla \cdot \mathbf{u}_{h}^{(n+1)}, q_{h}\right)+\left(\frac{C_{h}^{(n+1)}-C_{h}^{(n)}}{\Delta t}, \theta_{h}\right)+\left(\mathbf{u}_{h}^{(n)} \cdot \nabla C_{h}^{(n+1)}, \theta_{h}\right) \\
& +\left(a \nabla C_{h}^{(n+1)}, \nabla \theta_{h}\right)=\left(S, \theta_{h}\right) \\
& \quad \text { for } \mathbf{v}_{h} \in V_{h}, \quad q_{h} \in Q_{h}, \quad \theta_{h} \in \Theta_{h},
\end{aligned}
$$

where $\Delta t$ denotes a time increment. $\mathbf{u}_{h}^{(n)}, p_{h}^{(n)}$ and $C_{h}^{(n)}$ denote the finite element

approximations of $\mathbf{u}, p$ and $C$ at time $n \Delta t$, respectively. $V_{h}, Q_{h}$ and $\Theta_{h}$ are finite element approximation spaces of $V, Q$ and $\Theta$, respectively.

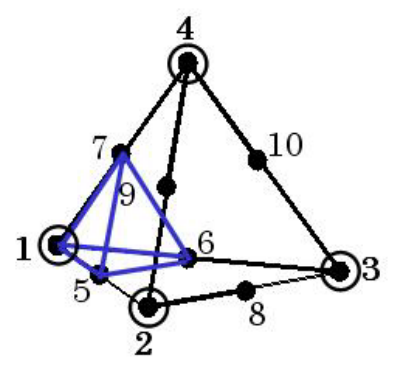

$\bigcirc$ pressure

- velocity and concentration

Fig.2 Bercovier-Pironneau elements.

\subsection{Stabilization Methods}

Now, our computational scheme with stabilization terms is introduced as follows;

$$
\begin{gathered}
B^{N S}\left(\mathbf{u}_{h}, p_{h}, C_{h} ; \mathbf{v}_{h}, q_{h}, \theta_{h}\right)+B^{C O}\left(\mathbf{u}_{h} ; \mathbf{v}_{h}, q_{h}\right)+B^{A D}\left(\mathbf{u}_{h}, C_{h} ; \mathbf{v}_{h}, \theta_{h}\right)=F^{A D}\left(\mathbf{v}_{h}, \theta_{h}\right) \\
\quad \operatorname{for}\left(\mathbf{v}_{h}, q_{h}, \theta_{h}\right) \in V_{h} \times Q_{h} \times \Theta_{h}
\end{gathered}
$$


where

$B^{N S}(\mathbf{u}, p, C ; \mathbf{v}, q, \theta)=\left(\frac{\mathbf{u}^{(n+1)}-\mathbf{u}^{(n)}}{\Delta t}, \mathbf{v}\right)+\left(\left(\mathbf{u}^{(n)} \cdot \nabla\right) \mathbf{u}^{(n+1)}, \mathbf{v}\right)+\left(2 v D\left(\mathbf{u}^{(n+1)}\right), D(\mathbf{v})\right)$ $-\left(p^{(n+1)}, \nabla \cdot \mathbf{v}\right)+\left(\beta C^{(n+1)} \mathbf{g}, \mathbf{v}\right)+\sum_{K \in \mathfrak{I}_{h / 2}} \tau_{K}^{N S}\left(\begin{array}{l}\frac{\mathbf{u}^{(n+1)}-\mathbf{u}^{(n)}}{\Delta t}+\left(\mathbf{u}^{(n)} \cdot \nabla\right) \mathbf{u}^{(n+1)} \\ +\nabla p^{(n+1)}+\beta C^{(n+1)} \mathbf{g}, \\ \left(\mathbf{u}^{(n)} \cdot \nabla\right) \mathbf{v}-\nabla q\end{array}\right)_{K}$

$B^{C O}(\mathbf{u} ; \mathbf{v}, q)=-\left(\nabla \cdot \mathbf{u}^{(n+1)}, q\right)+\sum_{K \in \mathfrak{I}_{h / 2}} \tau_{K}^{C O}\left(\nabla \cdot \mathbf{u}^{(n+1)}, \nabla \cdot \mathbf{v}\right)_{K}$,

$B^{A D}(\mathbf{u}, C ; \mathbf{v}, \theta)=\left(\frac{C^{(n+1)}-C^{(n)}}{\Delta t}, \theta\right)+\left(\mathbf{u}^{(n)} \cdot \nabla C^{(n+1)}, \theta\right)+\left(a \nabla C^{(n+1)}, \nabla \theta\right)$

$+\sum_{K \in \mathfrak{I}_{h / 2}} \tau_{K}^{A D}\left(\begin{array}{l}\frac{C^{(n+1)}-C^{(n)}}{\Delta t}+\mathbf{u}^{(n)} \cdot \nabla C^{(n+1)}, \\ \mathbf{u}^{(n)} \cdot \nabla \theta\end{array}\right)_{K}$,

$F^{A D}(\mathbf{v}, \theta)=(S, \theta)+\sum_{K \in \mathfrak{I}_{h / 2}} \tau_{K}^{A D}\left(S, \mathbf{u}^{(n)} \cdot \nabla \theta\right)_{K}$,

where $(\cdot, \cdot)_{K}$ denotes the $L^{2}$ - inner product over each subdivided tetrahedral closed piece $K$. The stabilization parameters $\tau_{K}^{N S}, \tau_{K}^{C O}$ and $\tau_{K}^{A D}$ are defined by

$$
\begin{aligned}
& \tau_{K}^{N S} \equiv \min \left\{\frac{\Delta t}{2}, \frac{h_{K}}{2\left|\mathbf{u}_{h}^{(n)}\right|_{\infty}}, \frac{h_{K}^{2}}{24 v}\right\}, \\
& \tau_{K}^{C O} \equiv \min \left\{\frac{\lambda\left|\mathbf{u}_{h}^{(n)}\right|_{\infty}^{2} h_{K}^{2}}{12 v}, \lambda\left|\mathbf{u}_{h}^{(n)}\right|_{\infty} h_{K}\right\}, \\
& \tau_{K}^{A D} \equiv \min \left\{\frac{\Delta t}{2}, \frac{h_{K}}{2\left|\mathbf{u}_{h}^{(n)}\right|_{\infty}}, \frac{h_{K}^{2}}{12 a}\right\} .
\end{aligned}
$$

where the constant $\lambda$ is set to be $1,|\mathbf{u}|_{\infty}$ denotes the maximum norm of $\mathbf{u}$ in $K$, and $h_{K}$ denotes the diameter of $K$. The symbol $\mathfrak{I}_{h / 2}$ denotes the subdivision of the triangulation $\mathfrak{I}_{h}$, which is constructed by using eight small tetrahedral pieces of each 
element of $\mathfrak{I}_{h}$.

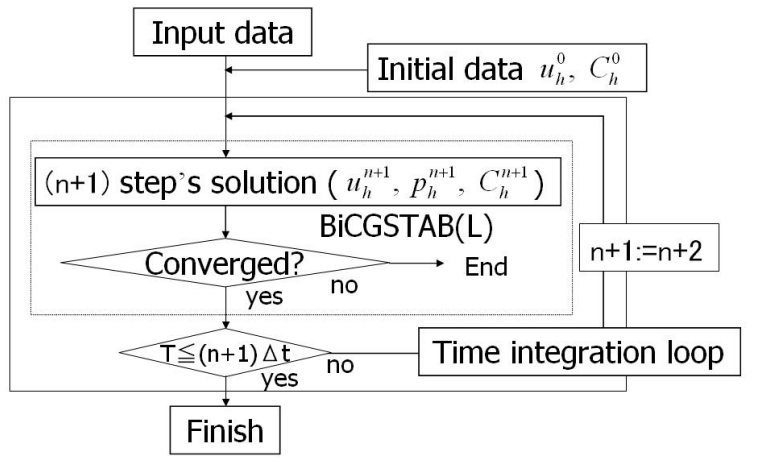

Fig.3 Analysis flow chart.

\section{Numerical Results}

\subsection{Parameters}

Several parameters including gas properties are summarized in Table $\mathbf{1 .}$

Table 1 Parameters.

\begin{tabular}{|c|c|}
\hline Parameters & Values \\
\hline kinematic viscosity $v$ & $1.05 \times 10^{-4}\left[\mathrm{~m}^{2} / \mathrm{s}\right]$ \\
\hline diffusion coefficient in air $a$ & $6.1 \times 10^{-5}\left[\mathrm{~m}^{2} / \mathrm{s}\right]$ \\
\hline coefficient $\beta$ & $13.4[-]$ \\
\hline gravity $g$ & $(0,0,-9.8)\left[\mathrm{m} / \mathrm{s}^{2}\right]$ \\
\hline source term $S$ & $0 \quad[1 / \mathrm{s}]$ \\
\hline
\end{tabular}

\subsection{The Coefficient $\beta$}

In thermal convection problems, the coefficient $\beta$ is known as the thermal expansion coefficient. But, in this hydrogen dispersion, the coefficient $\beta$ is unknown. We determine the coefficient $\beta$ by the following;

The buoyancy force is represented by;

$$
\mathbf{f}=\left(1-\frac{\rho_{\text {air }}}{\rho}\right) \mathbf{g},
$$

where $\mathbf{f}$ is the buoyancy force normalized by the density $\left[\mathrm{m} / \mathrm{s}^{2}\right] ; \quad \rho_{\text {air }}$ is the density of air $\left[\mathrm{kg} / \mathrm{m}^{3}\right] ; \rho$ is the mixture gas density $\left[\mathrm{kg} / \mathrm{m}^{3}\right]$ represented by;

$$
\rho=\frac{P_{m}}{\left[C R_{H_{2}}+(1-C) R_{\text {air }}\right] T_{m}},
$$


where $P_{m}$ is the absolute pressure of the mixture gas $[\mathrm{Pa}] ; T_{m}$ is the absolute temperature

$[\mathrm{K}] ; R_{H_{2}}$ is the gas constant of hydrogen $[\mathrm{J} /(\mathrm{kg} \cdot \mathrm{K})] ; R_{\text {air }}$ is the gas constant of air $[\mathrm{J} /(\mathrm{kg} \cdot \mathrm{K})]$

On the other hand, the buoyancy force in the Boussinesq approximation is represented by the following;

$$
\mathbf{f}=-\beta C \mathbf{g}
$$

We write Fig.4 using the following parameters in Table 2. Figure 4 shows the coefficient $\beta=13.4$.

Table 2 Parameters at $1[\mathrm{~atm}]$ and $20\left[{ }^{\circ} \mathrm{C}\right]$.

\begin{tabular}{|l|l|}
\hline \multicolumn{1}{|c|}{ Parameters } & \multicolumn{2}{|c|}{ Values } \\
\hline density of air $\rho_{\text {air }}$ & $1.209 \quad\left[\mathrm{~kg} / \mathrm{m}^{3}\right]$ \\
\hline gas constant of hydrogen $R_{H_{2}}$ & $4,122 \quad[\mathrm{~J} /(\mathrm{kg} \cdot \mathrm{K})]$ \\
\hline gas constant of air $\quad R_{\text {air }}$ & $287 \quad[\mathrm{~J} /(\mathrm{kg} \cdot \mathrm{K})]$ \\
\hline absolute temperature $T_{\mathrm{m}}$ & $293 \quad[\mathrm{~K}]$ \\
\hline absolute pressure $\quad P_{\mathrm{m}}$ & \multicolumn{2}{|c|}{$1.01 \times 10^{5}[\mathrm{~Pa}]$} \\
\hline
\end{tabular}

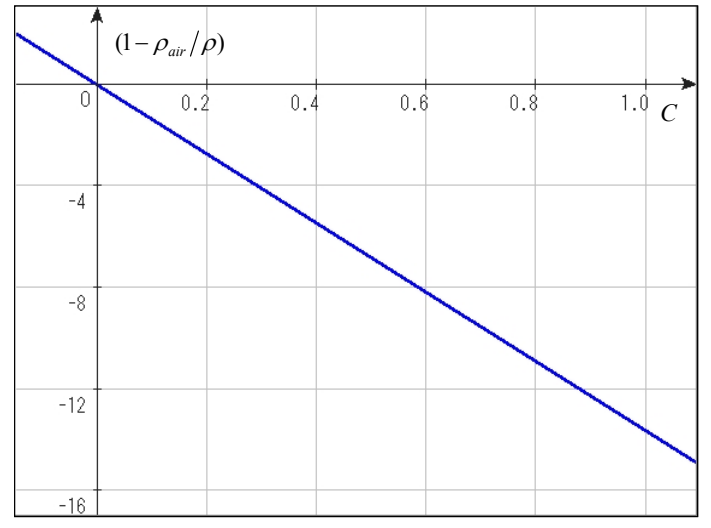

Fig.4 Relation between $C$ and $\left(1-\rho_{\text {air }} / \rho\right)$.

\subsection{Other Numerical Conditions}

The number of elements and degrees of freedom are 165,434 and 870,447, respectively. A time increment is $0.5[\mathrm{~s}]$. $\operatorname{BiCGSTAB}(L)$ method $^{6)}$ with the incomplete $\boldsymbol{L} \boldsymbol{D} \boldsymbol{U}$ factorization preconditioner is used as the solver for each step in the time integration. The number $L$ is set to be 4 . The acceleration factor is set to be 1.05. Computation of the model was performed on a Pentium $4(3.2 \mathrm{GHz})$ with $1 \mathrm{CPU}$. It took 70 hours for the computation. 


\subsection{Results}

Figure 5 shows our computational results of the hydrogen concentration at four sensors. We compare these results with the data of the reference ${ }^{7)}$. Figure 6 shows the reference data. Both our results and the data are almost similar. In fact, it is noted that experimental $\mathrm{data}^{7)}$ at four sensors (specially at Sensor 1) also have strong oscillations like Fig.7. Therefore, we can say that we have more accurate verification of computational results than that in the previous paper ${ }^{3)}$. In fact, in the previous paper ${ }^{3)}$, we had about 10 times higher volumetric concentration in spite of similar volumetric concentration distributions when $\mathrm{C}=100$ [mass\%] was given in (5). Other computational conditions were completely the same. This fact means that $\mathrm{C}=6.94$ [mass\%] in (5) is so important, which considers the density difference between air and hydrogen.

Figure 8 shows velocity fields at the $x_{2}-x_{3}$ surface on $x_{1}=0.37 \mathrm{~m}$. Figure 9 shows the hydogen concentration distributions at the same time.

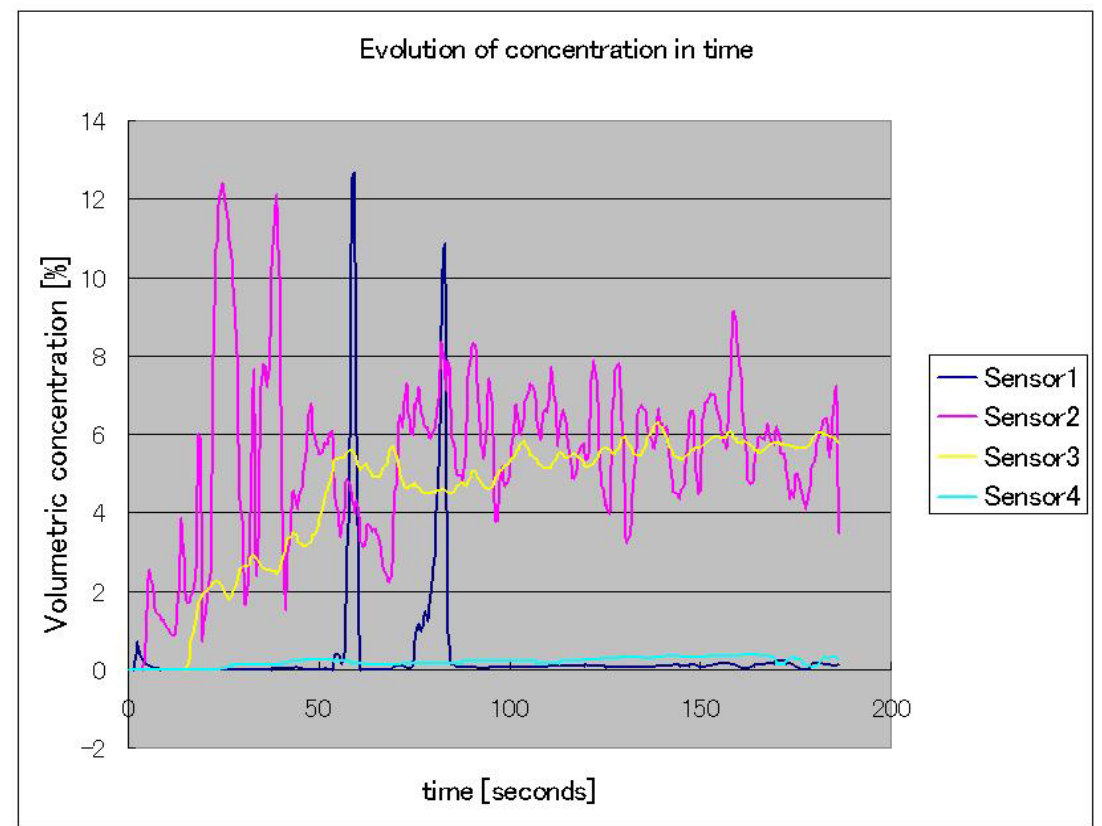

Fig. 5 Our results of the hydrogen concentration at four sensors.

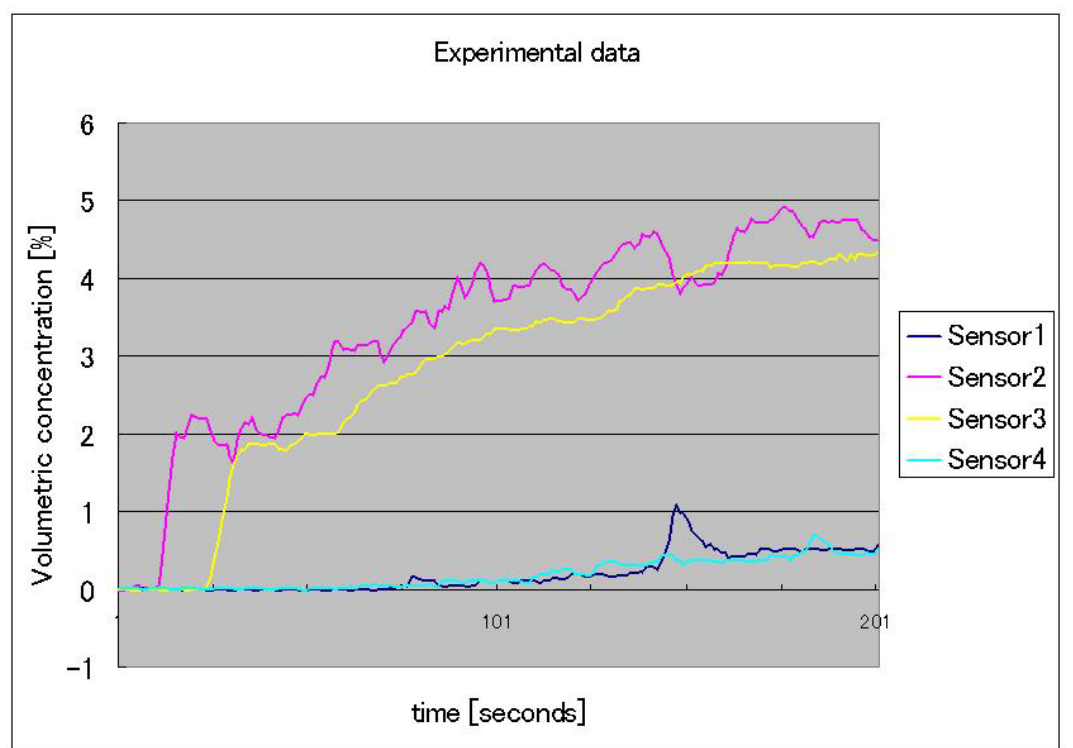

Fig. 6 Experimental data ${ }^{7)}$ 


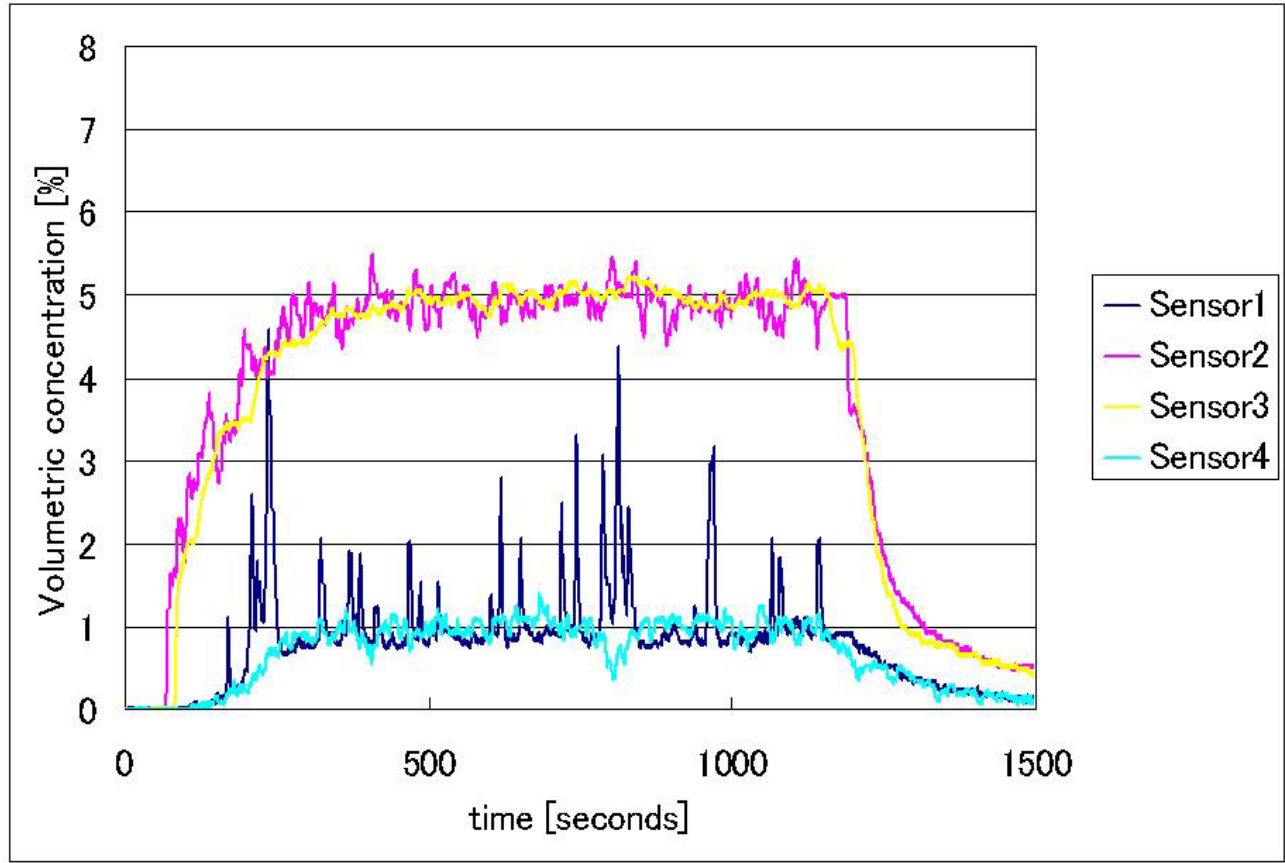

Fig. 7 Oscillations of experimental data ${ }^{7)}$

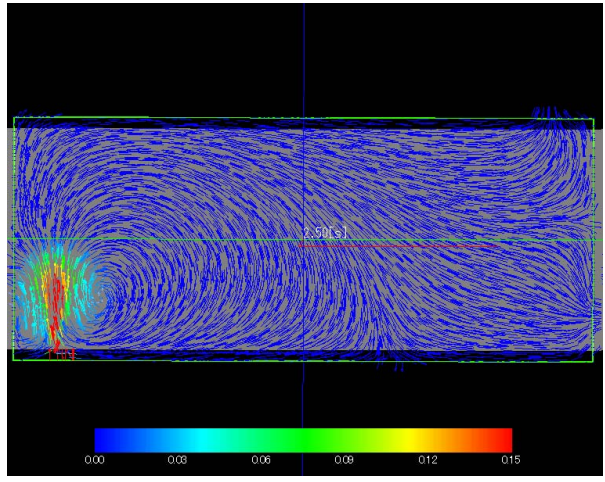

$2.5[\mathrm{~s}]$

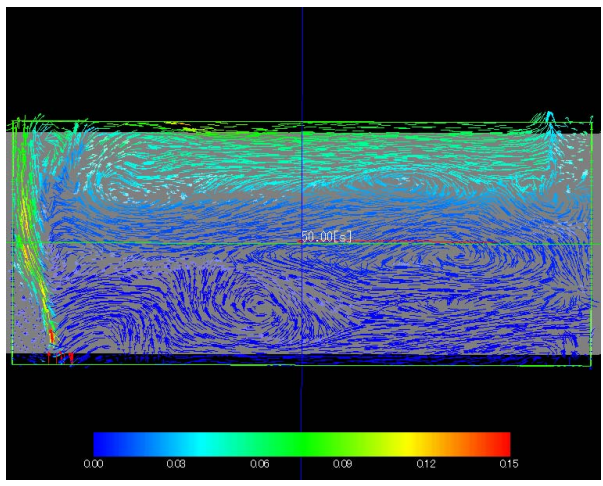

$50[s]$

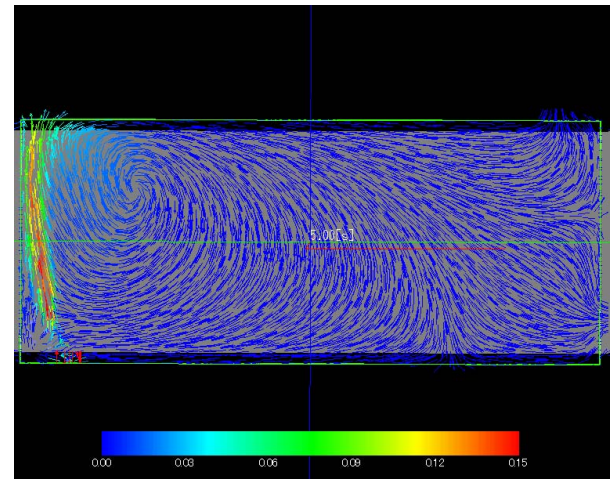

$5[\mathbf{s}]$

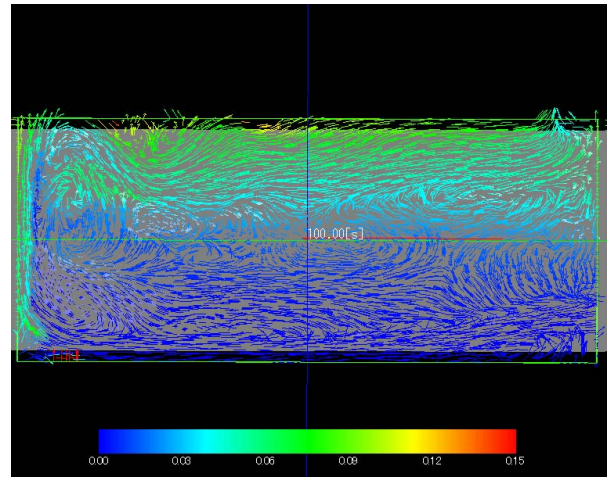

$100[s]$

Fig. 8 Velocity fields at time $2.5[\mathrm{~s}], 5[\mathrm{~s}], 50[\mathrm{~s}]$ and $100[\mathrm{~s}]$ (velocity range : $0 \sim 0.15[\mathrm{~m} / \mathrm{s}]$ ). 


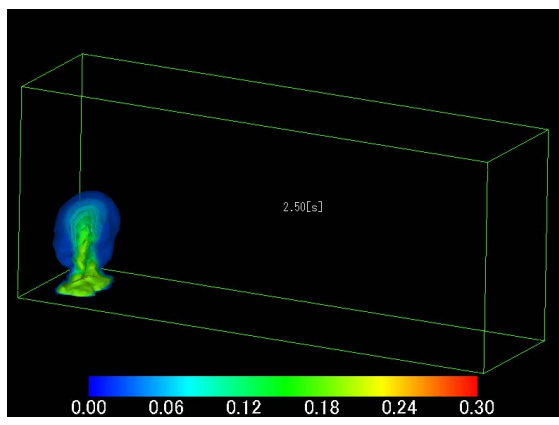

$2.5[\mathrm{~s}]$

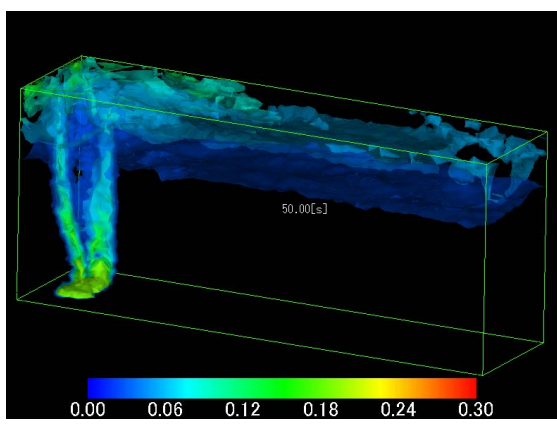

$50[s]$

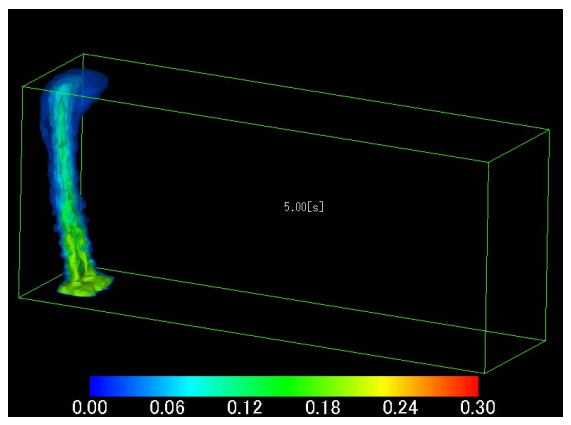

$5[\mathbf{s}]$

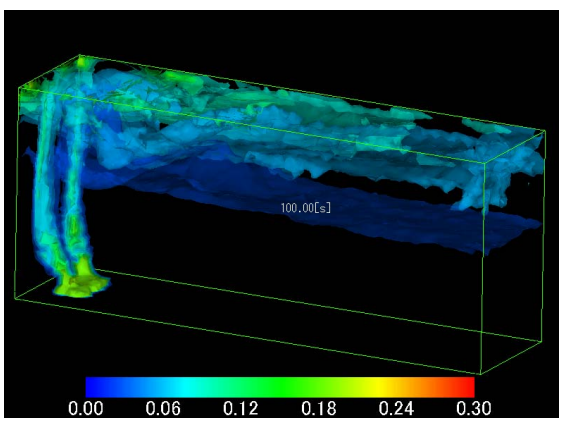

$100[s]$

Fig. 9 The hydrogen concentration distributions at time 2.5[s], 5[s], 50[s] and $100[\mathrm{~s}]$

(hydrogen concentration : 0 30 [volume \%]).

\section{Conclusions}

The dispersion phenomena of hydrogen are modeled by using the analogy of thermal convection problems with the Boussinesq approximation. The flow of hydrogen is grasped by using a stabilized finite element method. We have had more accurate verification of computational results than that in the previous paper ${ }^{3)}$.

\section{References}

(1) V. Agarant, Z. Cheng and A. Tchouvelev; CFD modeling of hydrogen releases and dispersion in hydrogen energy station, Proceedings of The 15th World Hydrogen Energy Conference, (2004).

(2) H. Kanayama, D. Tagami, T. Araki and H. Kume; A stabilization technique for steady flow problems, International Journal of Computational Fluid Dynamics, Vol.18, No.4, pp.297-301, May, (2004).

(3) H. Kanayama, K. Maeda, M. Mino and K. Matsuura; Finite element simulation of hydrogen dispersion, Computational Fluid Dynamics Journal, Vol. 15, No. 1, pp.101-106, (2006).

(4) D. Tagami and H. Itoh; A finite element analysis of thermal convection problems with the Joule heat, The Japan Journal of Industrial and Applied Mathematics, Vol. 20, No. 2, pp.193-210, (2003).

(5) M. Bercovier and O. Pironneau; Error estimates for finite element method solution of the Stokes problem in the primitive variables, Numerische Mathematik, 33, pp. 211-224, (1979).

(6) G.L.G. Sleijpen and D.R. Fokkema, BiCGSTAB (L) for linear equations involving unsymmetric matrices with complex spectrum, Electronic Transactions on Numerical Analysis, Vol. 1, pp.11-32, (1993).

(7) M. Inoue, H. Tsukikawa, H. Kanayama and K. Matsuura; Experimental study on leaking hydrogen dispersion in a partially open space, to appear in Journal of the Hydrogen Energy Systems Society of Japan, (2008) in Japanese. 